\title{
Clinical Significance of Improved Intraoperative Neurophysiological Monitoring Signal during Spine Surgery: A Retrospective Study of a Single- Institution Prospective Cohort
}

\author{
Seung Myung $\mathrm{Wi}^{1}$, Hui-Jong Lee ${ }^{2}$, Taehoon $\mathrm{Kang}^{2}$, Sam Yeol Chang ${ }^{2}$, Sung-Min Kim ${ }^{3}$, \\ Bong-Soon Chang ${ }^{2}$, Choon-Ki Lee ${ }^{2}$, Hyoungmin Kim² \\ ${ }^{1}$ Department of Orthopedic Surgery, Cheju Halla General Hospital, Cheju, Korea \\ ${ }^{2}$ Department of Orthopedic Surgery, Seoul National University Hospital, Seoul, Korea \\ ${ }^{3}$ Department of Neurology, Seoul National University Hospital, Seoul, Korea
}

Study Design: Retrospective case series.

Purpose: We reviewed the cases that showed significant improvement of intraoperative neurophysiological monitoring (IONM) signals during spine surgery to assess whether there is a correlation with signal improvement and postoperative clinical status and its clinical significance.

Overview of Literature: To reduce the risk of neural injury, many spine surgeons are using multimodality IONM. Although many studies attempted to identify valid alarm criteria for predicting postoperative neurologic deterioration, studies concerning the improvement of IONM signals are rare.

Methods: We reviewed all spine surgery cases with IONM data treated at our department between January 2013 and May 2017. We found cases showing significant IONM signal improvements. We prospectively analyzed the neurological and clinical outcomes of these patients and compared outcomes pre- and postoperatively.

Results: Among 317 cases with the IONM data, we found 29 cases that showed IONM signal improvement compared with baseline. There were 27 cases of compressive myelopathy: 22 had a degenerative cause at the cervical spine, and five, at the thoracic spine. There were two cases of huge neurogenic tumor each at the craniovertebral junction and at the lumbar spine. Both motor-evoked potentials (MEPs) and somatosensory-evoked potentials (SSEPS) signals were improved in six cases, only the MEPs signal improved in 10, and only SSEP signal improved in 13 cases. All cases showed the IONM signal improvement consistently after the decompression procedure during surgery. All patients had a significant improvement in neurological function and subjective symptoms, and none had neurologic deterioration postoperatively.

Conclusions: Improvement of IONM signals during surgery may indicate that no unrecognized neural injury occurred during surgery and a favorable postoperative neurological outcome can be expected.

Keywords: Spinal cord compression; Decompression; Intraoperative monitoring; Signal improvement; Neurophysiological monitoring

Received Jan 20, 2019; Revised Mar 11, 2019; Accepted May 8, 2019

Corresponding author: Hyoungmin Kim

Department of Orthopedic Surgery, Seoul National University Hospital, 101 Daehak-ro, Jongno-gu, Seoul 03080, Korea

Tel: +82-2-2072-0357, Fax: +82-2-764-2718, E-mail: hmkhm@snu.ac.kr 


\section{Introduction}

The development of neurological deficit following spinal surgery is a devastating complication. Some of these complications are known to occur without any recognizable adverse event during surgery. Recently, multimodality intraoperative neurophysiological monitoring (IONM), including somatosensory-evoked potentials (SSEPs), motor-evoked potentials (MEPs), and electromyography (EMG) has been utilized and has been proven effective for preventing neurological injury during spinal surgery $[1,2]$. SSEPs are used to monitor the dorsal column function, and MEPs monitor the function of the anterior and central portions of the spinal cord, including the corticospinal tract. Since the introduction of IONM, most studies have focused on signal loss as a sign of alarm indicating a neural injury, which may predict postoperative neurologic deterioration. Few studies have investigated the clinical meaning of an improved IONM signal during surgery [35]. Bouchard et al. [6] identified 11 patients with cervical spondylotic myelopathy who displayed intraoperative improvement of SSEP signals in 1996. Two additional studies, one by Visser et al. [7] and another by Wang et al. [8], reported that intraoperative MEPs significantly improved during surgical decompression of the spinal cord. Although these studies found a significant correlation between improved intraoperative signals and postoperative clinical outcome, both had several limitations. Only single MEP or SSEP modality was used rather than the modern multimodality IONM. Quantitative and objective evaluations of the patient's neurological status were not made, and thus pre- and postoperative comparisons could not be made. Thus, none of these studies adequately addressed a causal relationship between improved IONM signals and improved postoperative clinical outcomes.

To overcome the limitations of previous studies, we retrospectively reviewed cases that showed significant improvements in IONM signals to determine whether the improved signals correlated with the postoperative clinical status.

\section{Materials and Methods}

This study was approved by the institutional review board of the Seoul National University Hospital (IRB approval no., $\mathrm{H}-1707-003-864)$ and the Ethics Committee exempted the study for obtaining patient informed consent as this was a minimal risk study. We retrospectively reviewed 317 cases from a single-institution prospective cohort of patients who underwent spine surgery using multimodal IONM at the department of orthopedic surgery between January 2013 and May 2017. Of those, 29 patients, who showed an increase in IONM signals (MEPs or SSEPs) and were followed up for at least 6 months, were included in this study. No criteria have been established for what constitutes a significant improvement in IONM signals; therefore, we developed criteria based on the findings of previous studies. A significant MEP improvement was defined as a greater than $100 \%$ increase in amplitude or the appearance of a distinguishable waveform during surgery, and improvement in SSEP was defined as a greater than $100 \%$ increase in amplitude or decrease in latency of at least $6 \%[6,7]$.

\section{Anesthesia management}

The anesthetic regime was strictly controlled when the IONM was used. No premedication was administered. Patients received intravenous anesthetic agents (propofol 4.5 $\mu \mathrm{g} / \mathrm{mL}$ and/or remifentanil $1.7 \mathrm{ng} / \mathrm{mL}$ ) compatible with IONM, and short-acting muscle relaxants (rocuronium 0.8 $\mathrm{mg} / \mathrm{kg}$ ) were used for intubation but not during surgery. After intubation, appropriate reverse agents (glycopyrrolate $0.4 \mathrm{mg}$ and/or neostigmine $2 \mathrm{mg}$ ) were administered by an anesthesiologist.

\section{Multimodality intraoperative neurophysiological monitoring technique}

Multimodality IONM was performed by a trained surgical neurophysiologist using the NIM-ECLIPSE Spinal System (Medtronic Sofamor Danek, Memphis, TN, USA) according to the standard protocol. The procedure was successful in all patients. Serial monitoring was performed in both the lower and upper extremities from the time the patient was positioned on the operating table until the patient awakened from anesthesia. The stimulus amplitude was adjusted as needed for each patient. SSEPs were elicited by stimulation of the posterior tibial and median nerves. Cortical potentials were recorded from subdermal needle electrodes attached to standard cranial locations. Transcranial MEPs were recorded bilaterally from the deltoid, triceps, and thenar muscles in the upper extremities, and bilaterally from the tibialis anterior and abductor halluces 
muscles in the lower extremities. Spontaneous EMG was recorded at the same sites used to monitor MEPs. The neurophysiologist recorded the evoked discharges at baseline, after a change, at the time of the change, and during the main procedure. Then, the neurologist assessed the recordings as appropriate.

\section{Neurological and clinical outcome assessment}

Neurological assessment and patient-reported clinical outcome questionnaires were obtained pre- and postoperatively during regular follow-up visits to the outpatient clinic. For clinical analysis, preoperative data and at 3 months postoperatively were selected. Muscle strength was measured using the Motor Index Scoring System (MISS) (Table 1) [9]. Self-reporting questionnaires were used to assess the recovery of subjective symptoms: the Short-Form 36 Health Survey Questionnaire (SF-36) was administered to all patients [10]; the Japanese Orthopedic Association (JOA) Cervical Myelopathy Evaluation Questionnaire (JOACMEQ) and Neck Disability Index (NDI) were administered to patients with cervical myelopathy [11]; the JOA score was obtained for patients with thoracic myelopathy [12]; and the JOA Back Pain Evaluation Questionnaire [13]; and Oswestry Disability Index were administered to patients with lumbar disorders [14].

Table 1. Motor Index Scoring System

\begin{tabular}{lcc} 
& \multicolumn{2}{c}{ Motor grade } \\
\cline { 2 - 3 } Variable & Left muscle & Right muscle \\
\hline Shoulder abductors & 5 & 5 \\
Wrist extension & 5 & 5 \\
\hline Triceps & 5 & 5 \\
Grip & 5 & 5 \\
\hline Finger abduction & 5 & 5 \\
\hline Hip flexion & 5 & 5 \\
Knee extension & 5 & 5 \\
Ankle dorsiflexion & 5 & 5 \\
Extensor hallusis longus & 5 & 5 \\
Ankle plantar flexion & 5 & 5 \\
\hline Total (n=100) & 50 & 50 \\
\hline
\end{tabular}

$0=$ absent or total paralysis; $1=$ trace palpable or visible contraction; 2=poor, active ROM with gravity eliminated; $3=$ fair or active movement through ROM against gravity; 4=good or active movement through ROM against resistance; and $5=$ normal.

$\mathrm{ROM}$, range of motion.

\section{Statistical analysis}

All data are expressed as the mean \pm standard deviation. All statistical tests were performed using IBM SPSS software ver. 23.0 (IBM Corp., Armonk, NY, USA). Statistical comparisons were made using the Wilcoxon rank-sum test for comparing the data pre- and postoperatively. The Kruskal-Wallis test was used for comparing the improvement rate of MISS and SF-36. The threshold for significance was $p<0.05$.

\section{Results}

\section{Participants and descriptive data}

The IONM signal improved in 29 of 317 patients (9.1\%). Patient demographic characteristics are shown in Table 2. Both MEPs and SSEPs increased in five patients, MEPs increased in 10 patients, and SSEPs improved in 12 patients. The MEP amplitude increased more than 100\% in eight patients and a polyphasic waveform was elicited during surgery in one patient. Both are in one patient. SSEP amplitude increased more than $100 \%$ in 10 patients, and latency decreased in one patient. SSEP amplitude increased and latency decreased in one patient. The data of two patients who died from medical disorders unrelated to

Table 2. The demographics of patients

\begin{tabular}{lc} 
Characteristic & Value \\
\hline Age (yr) & $58.1 \pm 17.4$ (13-85) \\
Male:female & $23: 6$ \\
\hline Pathology (cases) & \\
$\quad$ Cervical myelopathy & 22 \\
\hline Neurofibroma, C1 (extradural) & 1 \\
\hline Thoracic myelopathy & 5 \\
\hline Neurogenic tumor, L5-S1 (extradural) & 1 \\
\hline Postoperative follow-up period (mo) & $13.1 \pm 5.6$ \\
\hline Surgical procedure & 13 \\
\hline Cervical laminoplasty & 1 \\
\hline Reduction \& arthrodesis (C1-2) & 5 \\
\hline Anterior cervical disc fusion & 8 \\
\hline Laminectomy & 2 \\
\hline Tumorectomy
\end{tabular}

Values are presented as mean \pm standard deviation (range), number of cases, or mean \pm standard deviation. 


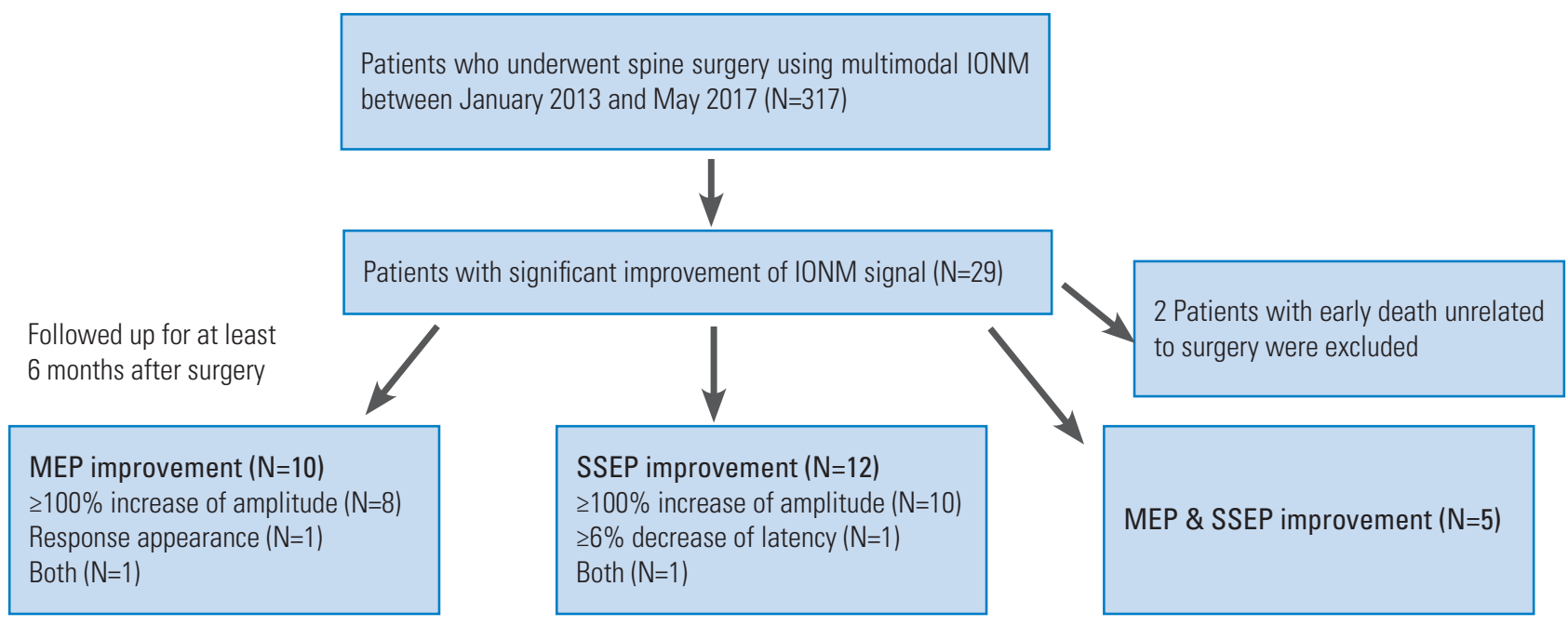

Fig. 1. Flow diagram for patient inclusion and distributions of IONM signal improvement patterns. IONM, intraoperative neurophysiological monitoring; MEP, motor-evoked potential; SSEP, somatosensory-evoked potential.

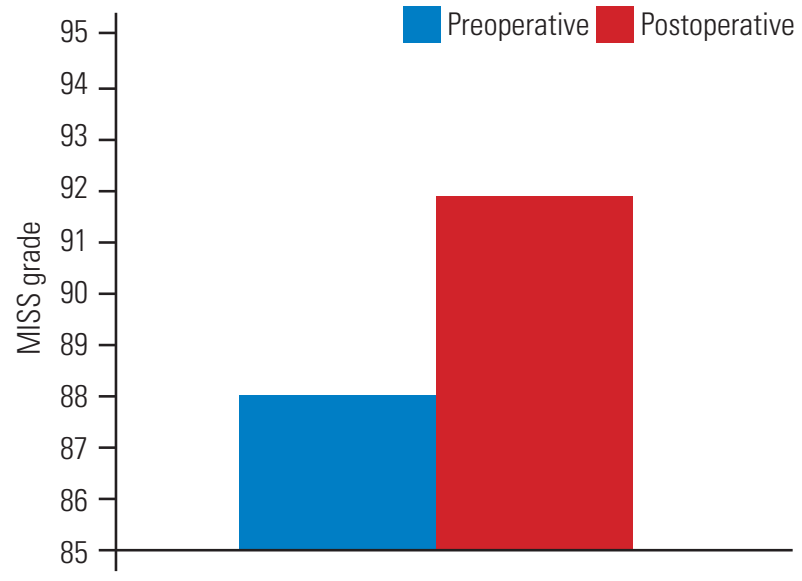

Fig. 2. MISS grade difference pre- and postoperatively. The mean preoperative MISS grade was $89.2 \pm 13.6$ which increased to $93.6 \pm 8.9$ postoperatively $(p<0.05)$. MISS, Motor Index Scoring System.

the surgery before postoperative month 2 were excluded from the analysis. A flow diagram of patient inclusion and distributions of IONM signal improvement patterns is provided in Fig. 1.

\section{Neurological and clinical outcome data}

The mean preoperative MISS score of $89.2 \pm 13.6$ increased to $93.6 \pm 8.9$ postoperatively $(p<0.05)$ (Fig. 2$)$. The MISS score did not decrease in any patient postoperatively. The pre- and postoperative MISS scores were not significantly different in seven patients, six of whom had no muscle weakness postoperatively. The results of the pre- and postoperative self-reporting questionnaires are shown in Table 3. The scores of SF-36, all JOACMEQ domains, with the exception of cervical spine function, the NDI, and the JOA significantly improved in cases of thoracic myelopathy compared to the preoperative values (SF-36, $102.6 \pm 8$ to $110.7 \pm 9.8$; JOACMEQ upper extremity function, $52.7 \pm 24.4$ to $77.4 \pm 23.8$; JOACMEQ lower extremity function, $21.1 \pm 16.7$ to $57.1 \pm 31.5$; JOACMEQ bladder function, $43.9 \pm 28$ to $62.1 \pm 33$.3; JOACMEQ quality of life, $21.6 \pm 16.6$ to $55.8 \pm 21.4$; NDI, $39.3 \pm 9$ to $23.8 \pm 9.8$; and JOA score for thoracic myelopathy, $5.8 \pm 1.6$ to $8.8 \pm 1.5 ; p<0.05$ ).

The MISS improvement rate in the MEP improvement group was significantly better than that in the other two groups $(p<0.05)$ and the SF-36 improvement rate in SSEP improvement group was significantly better than that in the other two groups $(p<0.05)$ (Table 4$)$.

\section{Illustrative case}

A 48-year-old male with ossification of the yellow ligament (OYL) at T11-12 presented with gait disturbance and paresthesia affecting both soles (Fig. 3A-C). Magnetic resonance imaging (MRI) and computed tomography images revealed severe central canal stenosis at the OYL site and compressive myelopathy. The patient could walk unaided, but he had a wide-based and spastic gait with a JOA score of 6. Surgical decompression of the spinal cord was performed as follows. Under general anesthesia, the patient's T11-12 spinous process and laminae were exposed via a standard midline spinal approach in a prone position. After total laminectomy of T11, the 
Table 3. Preoperative and postoperative patient-reported outcomes

\begin{tabular}{|c|c|c|c|}
\hline Questionnaire & Preoperative & Postoperative & $p$-value \\
\hline Short-Form 36 Health Survey Questionnaire & $102.6 \pm 8$ & $110.7 \pm 9.8$ & $<0.01$ \\
\hline \multicolumn{4}{|c|}{ JOA Cervical Myelopathy Evaluation Questionnaire } \\
\hline Cervical spine function & $43.8 \pm 31.9$ & $62.1 \pm 28.4$ & 0.06 \\
\hline Upper extremity function & $52.7 \pm 24.4$ & $77.4 \pm 23.8$ & $<0.01$ \\
\hline Lower extremity function & $21.1 \pm 16.7$ & $57.1 \pm 31.5$ & $<0.01$ \\
\hline Bladder function & $43.9 \pm 28$ & $62.1 \pm 33.3$ & $<0.01$ \\
\hline Quality of life & $21.6 \pm 16.6$ & $55.8 \pm 21.4$ & $<0.01$ \\
\hline Neck Disability Index & $39.3 \pm 9$ & $23.8 \pm 9.8$ & $<0.01$ \\
\hline JOA score & $5.8 \pm 1.6$ & $8.8 \pm 1.5$ & 0.04 \\
\hline \multicolumn{4}{|l|}{ JOA Back Pain Evaluation Questionnaire ( $n=1)$} \\
\hline Low back pain & 86 & 86 & \\
\hline Lumbar function & 92 & 100 & \\
\hline Walking ability & 100 & 100 & \\
\hline Social life function & 57 & 100 & \\
\hline Mental health & 59 & 97 & \\
\hline Oswestry Disability Index (n=1) & 16 & 9 & \\
\hline
\end{tabular}

Values are presented as mean \pm standard deviation or number.

JOA, Japanese Orthopedic Association.

Table 4. The patterns of significant intraoperative neurophysiological monitoring signal improvement and the improvement rate of MISS and SF-36

\begin{tabular}{lccc} 
Improvement pattern (cases) & $\begin{array}{c}\text { Motor-evoked potential } \\
\text { improvement group }(n=10)\end{array}$ & $\begin{array}{c}\text { Somatosensory-evoked potential } \\
\text { improvement group }(n=12)\end{array}$ & Both ( $n=5)$ \\
MISS improvement rate & $5.5 \% \pm 8.4 \%$ & $3.3 \% \pm 3.7 \%$ & $3.6 \% \pm 3.8 \%$ \\
SF-36 improvement rate & $8.2 \% \pm 10.4 \%$ & $8.8 \% \pm 7.7 \%$ & $6 \% \pm 10.7 \%$ \\
\hline
\end{tabular}

Values are presented as mean \pm standard deviation. Each improvement rate was calculated by the use of the following equation: (postoperative score-preoperative score)/(full score) $\times 100 \%$.

MISS, Motor Index Scoring System; SF-36, Short-Form 36 Health Survey Questionnaire.
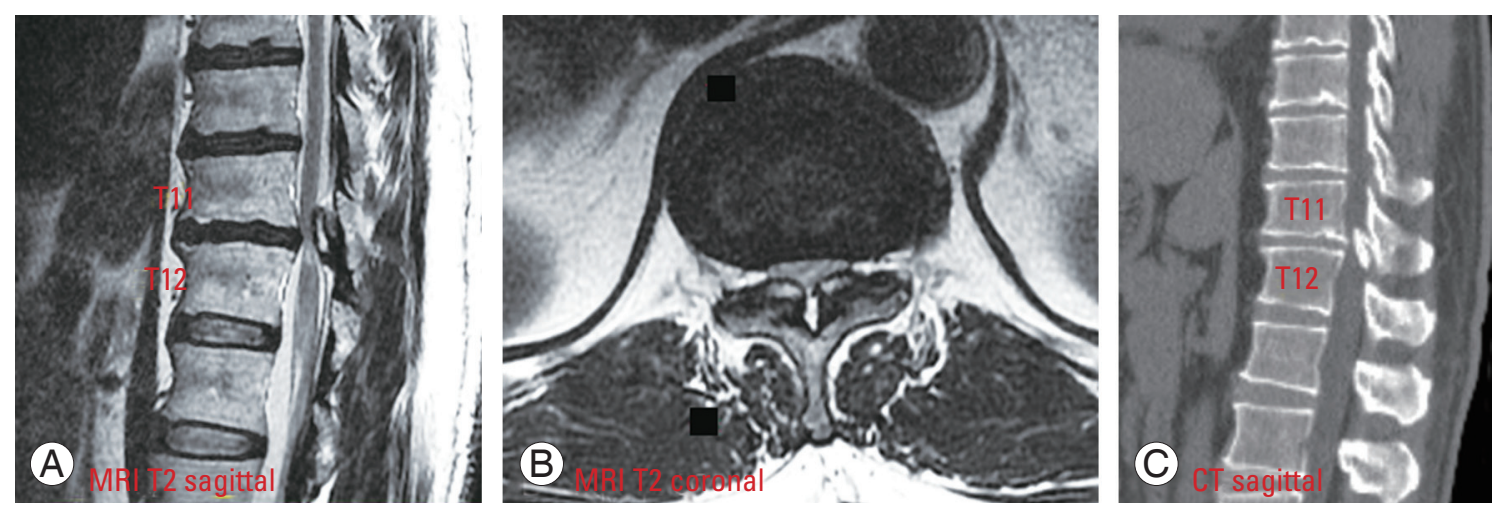

Fig. 3. (A-C) A 48-year-old man with OYL at T11-12 presented with gait disturbance and paresthesia affecting both soles. MRI and CT images revealed severe central canal stenosis at OYL location, leading to compressive myelopathy. He could walk unaided, but he stumbled and could not perform the tandem gait test. OYL, ossification of the yellow ligament; MRI, magnetic resonance imaging; CT, computed tomography. 

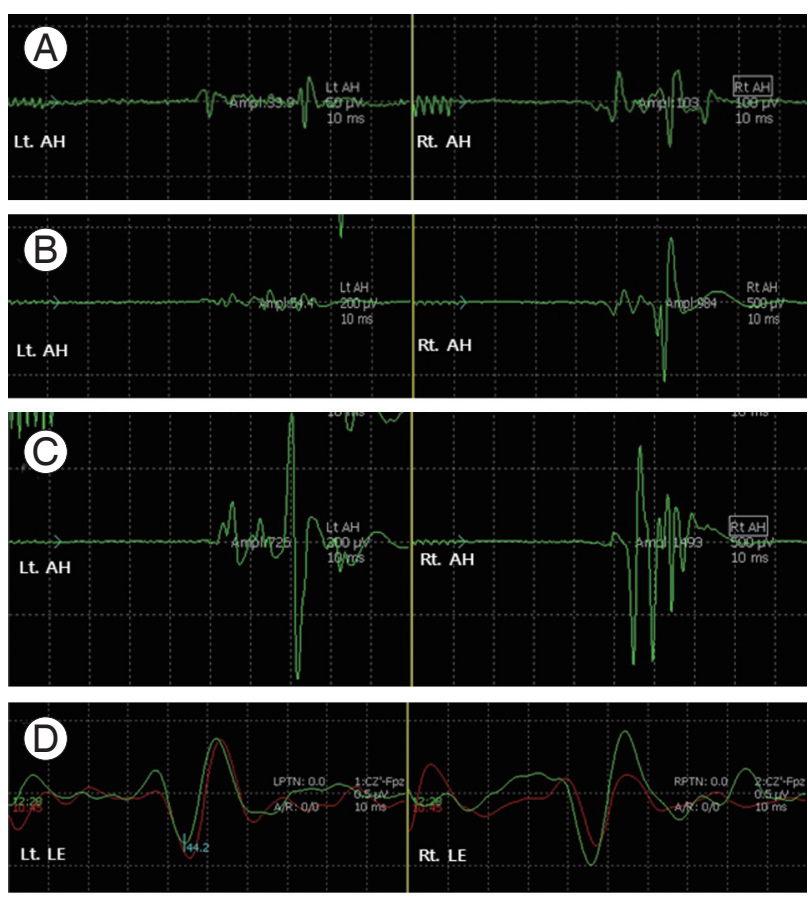

Fig. 4. Intraoperative MEP and SSEP recordings of patient 1 during the surgical procedure. (A) MEP recording before decompression. (B) MEP recording after right T11-12 laminectomy. (C) MEP recording after left T11-12 laminectomy. (D) SSEP recording at baseline (red) and after decompression (green). MEP, motor-evoked potential; SSEP, somatosensory-evoked potential; Lt, left; Rt, right; $\mathrm{AH}$, abductor halluces; LE, lower extremity.

bilateral OYL mass that was compressing the spinal cord was removed en bloc together with the superior articular process and proximal part of the T12 lamina. The MEPs and SSEPs during surgery for the patient are shown in Fig. $4 \mathrm{~A}-\mathrm{D}$. Following decompression on each side, the MEP amplitude improved sequentially. The amplitude of the right abductor halluces immediately increased from 103 $\mu \mathrm{V}$ to $984 \mu \mathrm{V}$ (Fig. 4A, B) after removal of the right OYL mass (Fig. 5A). Similarly, the amplitude of the left abductor halluces immediately increased from $50 \mu \mathrm{V}$ to $726 \mu \mathrm{V}$ (Fig. 4A, C) after removal of the left OYL mass (Fig. 5B). The red trace shows SSEPs at baseline, and the green trace shows SSEPs after decompression (Fig. 4D). The SSEP amplitude increased more than $100 \%$ after decompression in the right lower extremity. No postoperative neurological deficits occurred and his neurological status and paresthesia improved to a JOA score of 8 at the follow-up visit 6 months postoperatively.
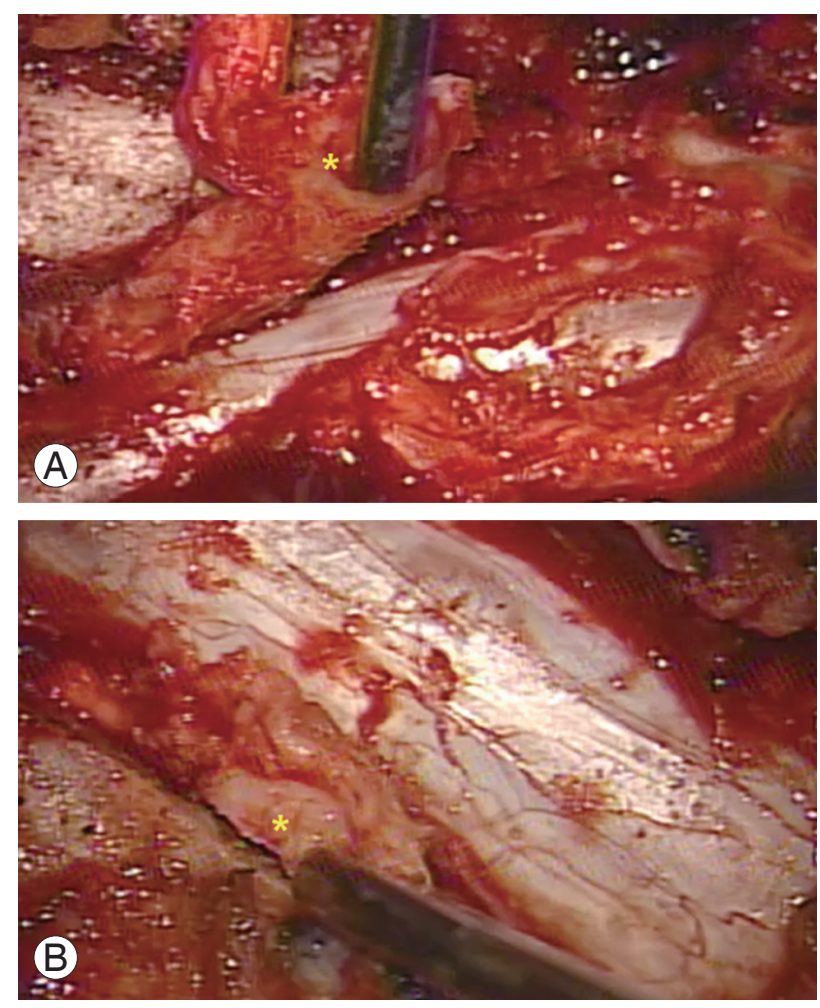

Fig. 5. Intraoperative microscopic images. Bilateral OYL mass that was compressing the spinal cord was removed en bloc together with the SAP and proximal part of the T12 lamina. (A) Removing right SAP and OYL mass $\left({ }^{*}\right)$ with a pituitary rongeur. Sequentially, the amplitude of the right abductor halluces immediately improved from $103 \mu \mathrm{V}$ to $984 \mu \mathrm{V}$ (Fig. 4A, B). (B) Removing left SAP and OYL mass (*) with a pituitary rongeur. Sequentially, the amplitude of the left abductor halluces immediately improved from $50 \mu \mathrm{V}$ to $726 \mu \mathrm{V}$ (Fig. 4A, C). OYL, ossification of the yellow ligament; SAP, superior articular process.

\section{Discussion}

We reviewed 29 cases, in which patients showed a significant improvement in the IONM signal after decompression of the spinal cord or cauda equina. We assessed each case using an objective manual muscle strength test, and self-reporting questionnaires were used to evaluate subjective symptoms. The neurological status was not aggravated postoperatively in any patient. Moreover, neurological function and subjective symptoms significantly improved postoperatively in all cases. Previous studies of IONM have reported similar findings; however, in addition to comparing pre- and postoperative neurological statuses, we assessed subjective symptoms and overall function, using various self-reporting questionnaires with proven validity and reliability for the different pathologies. Thus, our study provided a more objective assessment of the clinical symptoms and neurological status than previ- 
ous studies [6-8].

Many factors influence the neurological recovery after decompressive surgery for cervical and thoracic myelopathy. Previous studies have suggested that the surgical outcome and prognostic factors include patient clinical characteristics (age at surgery, sex, duration of symptoms, preoperative JOA score, type of myelopathy, radiological findings, spinal cord evoked potentials type, surgical procedure, and follow-up period duration), intramedullary signal change on MRI, and outcomes of the 15-second grip-and-release test and the 10-second step test [15-20]. However, the prognostic guidelines are not clear, thus, making it difficult for the surgeon to predict neurological recovery and outcome. Moreover, postoperative neurological deficits may occur even if no adverse event was observed during surgery. Our findings suggest that improved IONM signals predict a favorable surgical outcome and provide robust evidence that no adverse events occurred during surgery $[21,22]$. Consideration of IONM data together with the previously identified prognostic factors will enhance surgeons' ability to predict the postoperative outcome.

Several factors can affect the IONM signal. If there are changes in the IONM signal, a neural injury during surgery should be considered. Ischemic injury may decrease the amplitude, and a mechanical injury may delay the latency and decrease the amplitude. Notably, all inhaled anesthetics produce dose-dependent suppression of the amplitude, but intravenous anesthetics are compatible with multimodal IONM. As muscle relaxants block neurotransmission, there are used only for intubation. Blood pressure, body temperature, and oxygenation can also affect the IONM signal. Evoked potentials deteriorate when the body temperature is below $28^{\circ} \mathrm{C}$ and above $42^{\circ} \mathrm{C}$, and it has been suggested that evoked potentials should be performed within a range of $2^{\circ} \mathrm{C}-2.5^{\circ} \mathrm{C}$ above or below the baseline temperature. Hypocapnia affects SSEPs, and an abrupt drop in blood pressure may cause a change in the potential [23-25]. Furthermore, technical problems may also affect the signal. Our study included patients with various diagnoses; however, all etiologies involved the mechanical compression of neural tissue (i.e., cervical and thoracic spinal cord or cauda equina). Direct mechanical compression of the neural tissue and its blood supply are thought to cause neurological impairment $[26,27]$. In our study, IONM signals improved after the main procedure of spinal cord decompression in all patients. Other potential factors influencing signal were controlled within the authors' aforementioned protocol of the IONM. Therefore, it is reasonable to assume that the resolution of mechanical compression by surgical decompression improved nerve conduction and other potential explanations can be ruled out. The mean estimated blood loss was $246.7 \pm 191.3 \mathrm{~mL}$. The anesthetic regime was strictly controlled. The vital signs and arterial oxygen saturation were maintained within the normal range. Body temperature was maintained between $33.2^{\circ} \mathrm{C}$ and $36.4^{\circ} \mathrm{C}$. Furthermore, IONM was performed by a trained neurophysiologis who used a consistent and reproducible neuromonitoring technique.

Still, our study had several limitations. First, the retrospective design may be considered of low methodological quality. Second, our sample size was relatively small and included only one patient with a lumbar lesion, a massive intradural-extramedullary tumor. The low number of lumbar lesion cases can be explained by the fact that IONM was primarily used when managing high-risk spinal cord injury cases at our institution. However, our study included more cases showing improved IONM data than previously reported studies with a similar objective. Bouchard et al. [6] found that SSEPs improved in 11 patients. In a case series of patients with spinal cord compression, Visser et al. [7] reported that MEPs significantly improved in eight patients, and Wang et al. [8] found that intraoperative MEPs improved after cervical cord decompression in 21 patients. Finally, we did not compare the outcomes of patients who showed signal improvement with those of patients whose signals did not change or deteriorated during surgery. Additional studies are needed to investigate these outcomes and determine the correlation between IONM findings and preoperative clinical neurological abnormalities, such as pyramidal tract and dorsal column dysfunction.

\section{Conclusions}

We identified cases showing significant improvements in IONM signals and evaluated the correlation between intraoperative signal improvement and clinical outcomes. Improvement in IONM signals is a predictor of favorable neurological outcome, particularly for spinal cord decompression surgery. Based on the present findings, we are confident in that intraoperative neural injury can be ruled out in cases with improved IONM signals and that 
neurological function can be expected to improve postoperatively. Therefore, we urge surgeons to consider the improved IONM signals as a sign of successful decompression.

\section{Conflict of Interest}

No potential conflict of interest relevant to this article was reported.

\section{Author Contributions}

HJL, SYC, SMK, and THK were involved in the clinical management of these patients and collected clinical details of this study. SMW was responsible for patient selection and recruitment and drafting the manuscript; HMK (corresponding author) was responsible for designing the study, carrying out the surgical procedures, processing data and critically revising the manuscript; BSC was carrying out the surgical procedures; CKL revising the manuscript. All authors read and approved the final manuscript.

\section{References}

1. Sutter M, Eggspuehler A, Grob D, et al. The diagnostic value of multimodal intraoperative monitoring (MIOM) during spine surgery: a prospective study of 1,017 patients. Eur Spine J 2007;16 Suppl 2:S162-70.

2. Lall RR, Lall RR, Hauptman JS, et al. Intraoperative neurophysiological monitoring in spine surgery: indications, efficacy, and role of the preoperative checklist. Neurosurg Focus 2012;33:E10.

3. Sutter M, Eggspuehler A, Muller A, Dvorak J. Multimodal intraoperative monitoring: an overview and proposal of methodology based on 1,017 cases. Eur Spine J 2007;16 Suppl 2:S153-61.

4. Raynor BL, Bright JD, Lenke LG, et al. Significant change or loss of intraoperative monitoring data: a 25-year experience in 12,375 spinal surgeries. Spine (Phila Pa 1976) 2013;38:E101-8.

5. Gonzalez AA, Jeyanandarajan D, Hansen C, Zada G, Hsieh PC. Intraoperative neurophysiological monitoring during spine surgery: a review. Neurosurg Focus 2009;27:E6.

6. Bouchard JA, Bohlman HH, Biro C. Intraoperative improvements of somatosensory evoked potentials: correlation to clinical outcome in surgery for cervical spondylitic myelopathy. Spine (Phila Pa 1976) 1996;21:589-94.

7. Visser J, Verra WC, Kuijlen JM, Horsting PP, Journee HL. Recovery of TES-MEPs during surgical decompression of the spine: a case series of eight patients. J Clin Neurophysiol 2014;31:568-74.

8. Wang S, Tian Y, Wang C, et al. Prognostic value of intraoperative MEP signal improvement during surgical treatment of cervical compressive myelopathy. Eur Spine J 2016;25:1875-80.

9. Lucas JT, Ducker TB. Motor classification of spinal cord injuries with mobility, morbidity and recovery indices. Am Surg 1979;45:151-8.

10. Ware JE Jr, Sherbourne CD. The MOS 36-item shortform health survey (SF-36): I. conceptual framework and item selection. Med Care 1992;30:473-83.

11. Ohya J, Oshima Y, Oka H, et al. Patient satisfaction with posterior decompression surgery for cervical ossification of the posterior longitudinal ligament: prognostic radiographic factors and patient-reported outcomes for the effectiveness of surgical treatment. World Neurosurg 2016;96:272-9.

12. Onishi E, Yasuda T, Yamamoto H, Iwaki K, Ota S. Outcomes of surgical treatment for thoracic myelopathy: a single-institutional study of 73 patients. Spine (Phila Pa 1976) 2016;41:E1356-63.

13. Hashizume H, Konno S, Takeshita K, et al. Japanese orthopaedic association back pain evaluation questionnaire (JOABPEQ) as an outcome measure for patients with low back pain: reference values in healthy volunteers. J Orthop Sci 2015;20:264-80.

14. Fairbank JC, Couper J, Davies JB, O'Brien JP. The Oswestry low back pain disability questionnaire. Physiotherapy 1980;66:271-3.

15. Uchida K, Nakajima H, Takeura N, et al. Prognostic value of changes in spinal cord signal intensity on magnetic resonance imaging in patients with cervical compressive myelopathy. Spine J 2014;14:1601-10.

16. Hosono N, Takenaka S, Mukai Y, et al. Postoperative 24-hour result of 15-second grip-and-release test correlates with surgical outcome of cervical compression myelopathy. Spine (Phila Pa 1976) 2012;37:1283-7.

17. Ogawa Y, Yukawa Y, Morita D, Ito K, Machino M, Kato F. 10-Second step test for quantitative evaluation of the severity of thoracic compressive myelopathy. Spine (Phila Pa 1976) 2013;38:1405-8. 
18. Sanghvi AV, Chhabra HS, Mascarenhas AA, Mittal VK, Sangondimath GM. Thoracic myelopathy due to ossification of ligamentum flavum: a retrospective analysis of predictors of surgical outcome and factors affecting preoperative neurological status. Eur Spine J 2011;20:205-15.

19. Cho YE, Shin JJ, Kim KS, et al. The relevance of intramedullary high signal intensity and gadolinium (Gd-DTPA) enhancement to the clinical outcome in cervical compressive myelopathy. Eur Spine J 2011;20:2267-74.

20. Uchida K, Nakajima H, Sato R, et al. Multivariate analysis of the neurological outcome of surgery for cervical compressive myelopathy. J Orthop Sci 2005;10:564-73.

21. Fehlings MG, Smith JS, Kopjar B, et al. Perioperative and delayed complications associated with the surgical treatment of cervical spondylotic myelopathy based on 302 patients from the AOSpine North America Cervical Spondylotic Myelopathy Study. J Neurosurg Spine 2012;16:425-32.

22. Ito Z, Matsuyama $Y$, Ando M, et al. Postoperative paralysis from thoracic ossification of posterior lon- gitudinal ligament surgery risk factor of neurologic injury: nationwide multiinstitution survey. Spine (Phila Pa 1976) 2016;41:E1159-63.

23. Pajewski TN, Arlet V, Phillips LH. Current approach on spinal cord monitoring: the point of view of the neurologist, the anesthesiologist and the spine surgeon. Eur Spine J 2007;16 Suppl 2:S115-29.

24. Owen JH. The application of intraoperative monitoring during surgery for spinal deformity. Spine (Phila Pa 1976) 1999;24:2649-62.

25. Noonan KJ, Walker T, Feinberg JR, Nagel M, Didelot W, Lindseth R. Factors related to false- versus truepositive neuromonitoring changes in adolescent idiopathic scoliosis surgery. Spine (Phila Pa 1976) 2002;27:825-30.

26. Lips J, de Haan P, de Jager SW, Vanicky I, Jacobs MJ, Kalkman CJ. The role of transcranial motor evoked potentials in predicting neurologic and histopathologic outcome after experimental spinal cord ischemia. Anesthesiology 2002;97:183-91.

27. Bohlman HH, Emery SE. The pathophysiology of cervical spondylosis and myelopathy. Spine (Phila Pa 1976) $1988 ; 13: 843-6$. 